Research Paper

\title{
Exploration and Validation of C-Reactive Protein/ Albumin Ratio as a Novel Inflammation-Based Prognostic Marker in Nasopharyngeal Carcinoma
}

\author{
Yuan Zhang ${ }^{*}$, Guan-Qun Zhou1 ${ }^{*}$, Xu Liu1 ${ }^{*}$, Lei Chen ${ }^{1}$, Wen-Fei Li1, Ling-Long Tang1, Qing Liu², Ying Sun1, \\ Jun $\mathrm{Ma}^{1 凶}$ \\ 1. Department of Radiation Oncology, Sun Yat-sen University Cancer Center, State Key Laboratory of Oncology in South China, Collaborative Innovation \\ Center for Cancer Medicine, Canton, Guangdong Province, People's Republic of China. \\ 2. Department of Medical Statistics and Epidemiology, School of Public Health, Sun Yat-sen University, Canton, People's Republic of China. \\ * These authors contributed equally to this work. \\ $\square$ Corresponding author: Prof. Jun Ma, M.D., Department of Radiation Oncology, Sun Yat-sen University Cancer Center, 651 Dongfeng Road East, Canton \\ 510060, People's Republic of China. Telephone: +86-20-87343469 Fax: +86-20-87343295 E-mail: majun2@mail.sysu.edu.cn.
}

( ) Ivyspring International Publisher. Reproduction is permitted for personal, noncommercial use, provided that the article is in whole, unmodified, and properly cited. See http://ivyspring.com/terms for terms and conditions.

Received: 2016.02.28; Accepted: 2016.05.18; Published: 2016.07.04

\begin{abstract}
Background: The prognostic value of C-reactive protein/albumin ratio (CRP/Alb), a novel inflammation-based marker, remains unknown in nasopharyngeal carcinoma (NPC). Methods: We conducted a retrospective review of 1572 consecutive patients with non-metastatic NPC. Patients were randomly divided into a training set $(n=514)$ and validation set $(n=1058)$. The prognostic value of the CRP/Alb ratio and the modified Glasgow prognostic score (mGPS; a well-recognized inflammation-based score) was assessed. Results: Receiver-operating characteristic analysis identified 0.05 as the optimal CRP/Alb cut-off value for disease failure in the training set. Patients with a CRP/Alb $>0.05$ had poorer overall survival (OS), distant metastasis-free survival (DMFS) and disease-free survival (DFS) in the training set (all $P<0.05$ ). These results were confirmed in the validation set (all $P<0.05$ ) and the whole cohort (all $P<$ 0.001 ). In multivariate analysis of the entire cohort, the pretreatment CRP/Alb ratio was an independent prognostic factor for OS (HR, 1.394; 95\% Cl, 1.004-1.937; $P=0.048)$ and DMFS (HR, $1.545 ; 95 \% \mathrm{Cl}, 1.124-2.122 ; P=0.007)$, but not for DFS $(P=0.083)$. The $\mathrm{mGPS}$ had no significant independent prognostic value for any end-point. Conclusion: CRP/Alb ratio is an useful prognostic indicator in patients with NPC, independent of disease stage.
\end{abstract}

Key words: C-reactive protein; albumin; modified Glasgow prognostic score; nasopharyngeal carcinoma; prognosis.

\section{Introduction}

Nasopharyngeal carcinoma (NPC) is prevalent in southern China, Southeast Asia, North Africa, the Middle East and Alaska [1,2]. Radiotherapy is the primary treatment modality for non-metastatic NPC and chemoradiotherapy is recommended for loco-regionally advanced disease [3-6]. Improvements in radiation techniques due to the wide-spread adoption of intensity-modulated radiotherapy (IMRT) instead of 2D conventional radiotherapy have significantly improved locoregional control [7-10], and distant metastasis is currently the major failure pattern in NPC $[6-9,11]$.

The tumor, node, metastasis staging system is currently the most reliable tool for determining clinical treatment strategies and predicting the outcome of patients. A number of other clinical factors and biological markers, including the gross tumor volume [12], apparent diffusion coefficient (ADC) [13] and lactate dehydrogenase levels (LDH) [14], have also been demonstrated to provide additional 
prognostic information in NPC.

Inflammation plays an important role in carcinogenesis and tumor progression; for example, inflammation has been reported to promote tumor cell survival, proliferation, migration and invasion and also stimulate angiogenesis [15-19]. Thus, the identification of inflammation-related biomarkers has attracted much interest in oncology research. Recently, a novel inflammation-based prognostic score, the C-reactive protein/albumin ratio (CRP/Alb), was shown to have prognostic value in predicting survival in hepatocellular carcinoma [20]. However, the prognostic value of the $\mathrm{CRP} / \mathrm{Alb}$ ratio in NPC has not yet been explored. In this study, we performed a large scale cohort analysis to investigate the prognostic value of the CRP/Alb ratio in patients with NPC. We also assessed the prognostic value of the modified Glasgow prognostic score (mGPS), which is a well-recognized inflammation-based score that is also based on the levels of CRP and albumin.

\section{Patients and methods}

\section{Patients}

This retrospective study was approved by the Institutional Committee for Clinical Studies and the requirement for informed consent was waived. From November 2009 to February 2012, a total of 1572 consecutive patients with histologically-proven NPC were included in this study. The mean age of the entire cohort was 45 years (range, 14-78 years), with a male-to-female ratio of 2.9:1.

All patients underwent a pretreatment evaluation including a complete patient history, physical examination, hematology and biochemistry profiles, MRI of the neck and nasopharynx, chest radiography, abdominal sonography, and a whole body bone scan using single photon emission computed tomography. Furthermore, positron emission tomography-computed tomography (PET-CT) was performed on 470/1572 (29.9\%) patients. Medical records and imaging studies were analyzed retrospectively, and all patients were staged according to the 7th edition of the International Union against Cancer/American Joint Committee on Cancer (UICC/AJCC) system. The characteristics of the patients analyzed in this study are summarized in Table 1.

\section{Treatment}

All patients were treated with IMRT; details of the techniques used at our center have been reported previously [10]. In total, 94.1\% (1094/1572) of patients with stage III-IV NPC received concurrent chemoradiotherapy \pm neoadjuvant/adjuvant chemotherapy in conjunction with a platinum-based therapeutic clinical trial. When possible, salvage treatments such as brachytherapy, neck dissection and/or chemotherapy were provided in the event of documented relapse or persistent disease.

Patients were examined at least every 3 months during the first 2 years, and thereafter, a follow-up examination was performed every 5 months during years 3-5 or until death. Distant failure was defined as the presence of metastases in locations beyond the regional nodes to which the cancer spread via vascular or lymphatic channels, such as the liver, bones or mediastinal lymph nodes. Any residual disease in the nasopharynx or cervical nodes 6 months after completion of radiotherapy was regarded as local relapse or regional relapse, respectively. [21]

Table 1. Clinicopathological characteristics of the 1572 patients with NPC.

\begin{tabular}{|c|c|c|c|}
\hline Characteristics & $\begin{array}{l}\text { Training Set }(\mathrm{n} \\
=514)\end{array}$ & $\begin{array}{l}\text { Validation set } \\
(\mathrm{n}=1058)\end{array}$ & $P$ \\
\hline Sex & & & 0.579 \\
\hline Male & $388(75.5 \%)$ & $784(74.1 \%)$ & \\
\hline Female & $126(24.5 \%)$ & $274(25.9 \%)$ & \\
\hline Age (years) & & & 0.071 \\
\hline$\leq 50$ & $341(66.3 \%)$ & $750(70.9 \%)$ & \\
\hline$>50$ & $173(33.7 \%)$ & $308(29.1 \%)$ & \\
\hline Histological type a & & & 0.735 \\
\hline Keratinizing carcinoma & $4(0.8 \%)$ & $5(0.5 \%)$ & \\
\hline Non-keratinizing carcinoma & & & \\
\hline differentiated & $29(5.6 \%)$ & $57(5.4 \%)$ & \\
\hline undifferentiated & $481(93.6 \%)$ & $996(94.1 \%)$ & \\
\hline T-category $\mathrm{b}$ & & & 0.068 \\
\hline $\mathrm{T} 1$ & $101(19.6 \%)$ & $183(17.3 \%)$ & \\
\hline $\mathrm{T} 2$ & $72(14.0 \%)$ & $166(15.7 \%)$ & \\
\hline $\mathrm{T} 3$ & $230(44.7 \%)$ & $527(49.8 \%)$ & \\
\hline $\mathrm{T} 4$ & $111(21.6 \%)$ & $182(17.2 \%)$ & \\
\hline $\mathrm{N}$-category $\mathrm{b}$ & & & 0.773 \\
\hline No & $77(15.0 \%)$ & $192(18.1 \%)$ & \\
\hline N1 & $321(62.5 \%)$ & $597(56.4 \%)$ & \\
\hline N2 & $71(13.8 \%)$ & $172(16.3 \%)$ & \\
\hline N3a & $12(2.3 \%)$ & $20(1.9 \%)$ & \\
\hline $\mathrm{N} 3 \mathrm{~b}$ & $32(6.4 \%)$ & $78(7.3 \%)$ & \\
\hline Stage ${ }^{b}$ & & & 0.243 \\
\hline I & $24(4.7 \%)$ & $65(6.1 \%)$ & \\
\hline II & $108(21.0 \%)$ & $213(20.1 \%)$ & \\
\hline III & $235(45.7 \%)$ & $518(49.0 \%)$ & \\
\hline IV & $147(28.6 \%)$ & $262(24.8 \%)$ & \\
\hline Chemotherapy & & & 0.073 \\
\hline Yes & $455(88.5 \%)$ & $900(85.1 \%)$ & \\
\hline No & $59(11.5 \%)$ & $158(14.9 \%)$ & \\
\hline
\end{tabular}

Evaluations during follow-up included a complete patient history, physical examination, hematology and biochemistry profiles, MRI of the neck and nasopharynx, chest radiography, abdominal sonography and a whole body bone scan. Distant 
metastases were diagnosed by clinical symptoms, physical examinations and imaging methods that included chest radiography, a bone scan, MRI, CT and/or abdominal sonography. All local recurrences were diagnosed by fiber-optic endoscopy and biopsy or MRI (or both) of the nasopharynx and the skull base showing progressive bone erosion and soft tissue swelling. Regional recurrences were diagnosed by clinical examination of the neck and, in doubtful cases, by fine needle aspiration or a MRI scan of the neck. [22]

\section{Statistical analysis}

Statistical analyses were performed using SPSS version 22.0 (IBM Corporation, Armonk, NY, USA). The independent samples $t$-test was used to examine the differences in continuous variables between groups. The 1572 patients with NPC were randomly divided into a training set $(n=514)$ and a validation set $(n=1058)$. Categorical variables were compared using the Chi-square test (or Fisher's exact test, if the expected number was less than five in at least one cell). Area under the receiver-operating characteristic (ROC) curves were used to select the optimal cut-off value for the pretreatment $\mathrm{CRP} / \mathrm{Alb}$ ratio in the training set by maximizing the conditional Youden score, based on the method described by Hanley and McNeil [23] and Zweig and Campbell [24].

The endpoints included overall survival (OS), distant metastasis-free survival (DMFS), locoregional relapse-free survival (LRRFS) and disease-free survival (DFS). OS, DMFS and LRRFS were calculated from the first day of treatment to death, the first distant metastasis or locoregional relapse, respectively. DFS was defined as the latency to the date of disease progression or death from any cause. Actuarial rates were calculated using the Kaplan-Meier method and compared using the log-rank test. A Cox proportional hazards model was used to test the independent significance of different factors by backward elimination of insignificant variables.[25] Host factors (age and gender), tumor factors (pathology type, $\mathrm{T}$ and $\mathrm{N}$ classification) and treatment method (radiotherapy alone vs. chemoradiotherapy) were included in all analyses. The criterion for statistical significance was set at $\alpha=$ 0.05 and all $P$-values were based on two-sided tests.

\section{Results}

\section{Patterns of treatment failure}

The median duration of follow-up for the entire cohort was 50.0 months (range, 1.4-76.4 months). A total of $163 / 1572(10.4 \%), 81 / 1572(5.2 \%)$ and $72 / 1572$ $(4.6 \%)$ patients developed distant metastases, local relapses and regional relapses, respectively; 150/1572
(9.5\%) patients died and 34/1572 (2.2\%) patients experienced both distant metastases and local-regional relapses. The 3-year OS, DMFS, LRRFS and DFS rates for the entire cohort were $94.0 \%, 90.8 \%$, $92.7 \%$ and $84.8 \%$, respectively.

\section{Pretreatment CRPIAlb ratio and tumor staging}

The median and mean pretreatment $\mathrm{CRP} / \mathrm{Alb}$ ratios for the entire cohort of 1572 patients with NPC were 0.036 and 0.093 , respectively (range, 0.002-4.594). Patients with Stage III-IV disease had a significantly higher pretreatment $\mathrm{CRP} / \mathrm{Alb}$ ratio (mean, 0.106; median, 0.043; interquartile range, 0.019-0.093) than patients with Stage I-II disease (mean, 0.057; median, 0.024; interquartile range, $0.011-0.050 ; P<0.001)$.

\section{Prognostic value of the pretreatment CRP/Alb ratio in the training set, validation set and entire cohort}

In the training set $(n=514)$, the optimal pretreatment $\mathrm{CRP} / \mathrm{Alb}$ ratio cut-off value with respect to disease failure was 0.05 . This value had the maximum area under the ROC curve and was used to classify the patients in the training set into the $\mathrm{CRP} / \mathrm{Alb}_{\text {high }}(>0.05)$ and CRP/Alb $\mathrm{Alow}(\leq 0.05)$ groups. As shown in Figure 1, The CRP/Alb high group had significantly poorer 3 -year OS $(89.5 \%$ vs. $97.4 \%, P<$ $0.001)$, DMFS ( $85.4 \%$ vs. $93.4 \%, P=0.003)$ and DFS $(78.6 \%$ vs. $87.6 \%, P=0.007)$ than the $C R P / A_{1 b}$ low group. However, there was no significant difference in 3-year LRRFS (90.2\% vs. $93.4 \%, P=0.521)$ between the $\mathrm{CRP} / \mathrm{Alb}_{\text {high }}$ and $\mathrm{CRP} / \mathrm{Alb}_{\text {low }}$ groups in the training set.

In the validation set $(n=1058)$, the 3 -year OS, DMFS, LRRFS and DFS rates for the CRP/Alb high group and CRP/ $\mathrm{Alb}_{\text {low }}$ group were $93.4 \%$ vs. $94.2 \%(P$ $=0.031), 88.2 \%$ vs. $93.0 \%(P=0.002), 92.6 \%$ vs. $93.1 \%$ $(P=0.448)$ and $82.4 \%$ vs. $87.0 \%(P=0.012)$, respectively (Figure 2). Similarly, in the entire cohort ( $n=1572)$, the CRP/Alb $b_{\text {high }}$ group had significantly poorer 3-year OS (92.1\% vs. 95.2\%, $P<0.001)$, DMFS (87.3\% vs. $93.1 \%, P<0.001)$ and DFS $(81.1 \%$ vs. $87.2 \%$, $P<0.001)$ than the CRP/Alb low group. Similarly to the training set, there was no difference in the 3-year LRRFS rates $(91.8 \%$ vs. $93.2 \%, P=0.315)$ between the $\mathrm{CRP} / \mathrm{Alb}$ high and CRP/Alb low groups in the entire cohort (Figure 3).

Multivariate analysis of the entire cohort $(n=$ 1572) was performed to adjust for confounding factors. The pretreatment $\mathrm{CRP} / \mathrm{Alb}$ ratio was identified as an independent prognostic factor for OS (HR, 1.394; 95\% CI, 1.004-1.937; $P=0.048)$ and DMFS (HR, 1.545; 95\% CI, 1.124-2.425; $P=0.007$ ), but not for DFS (HR, 1.235; 95\% CI, 0.973-1.568; $P=0.083$ ). 
A

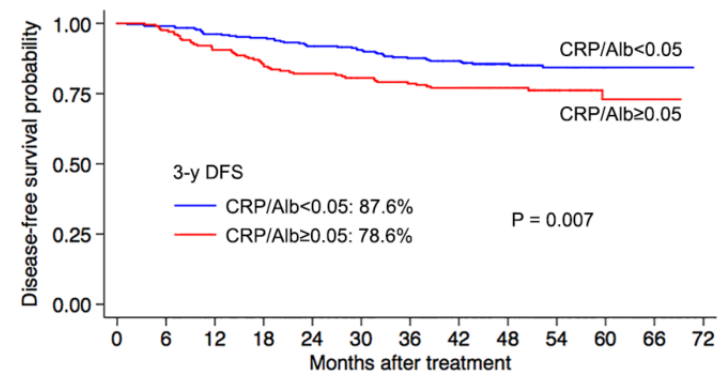

Number at risk

$\begin{array}{llllllllllllll}\text { CRP/Alb }<0.05 & 311 & 308 & 297 & 289 & 280 & 274 & 261 & 252 & 177 & 102 & 41 & 11 & 0\end{array}$ $\begin{array}{llllllllllllll}\text { CRP/Alb } \geq 0.05 & 203 & 196 & 182 & 172 & 165 & 160 & 153 & 149 & 119 & 57 & 21 & 3 & 0\end{array}$

C

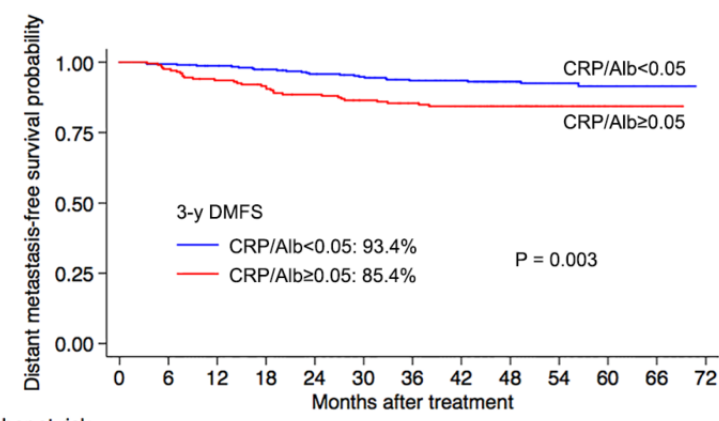

Number at risk

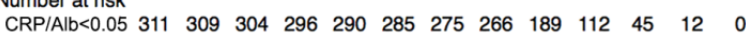

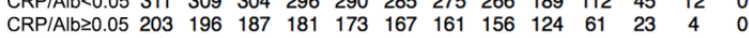

B

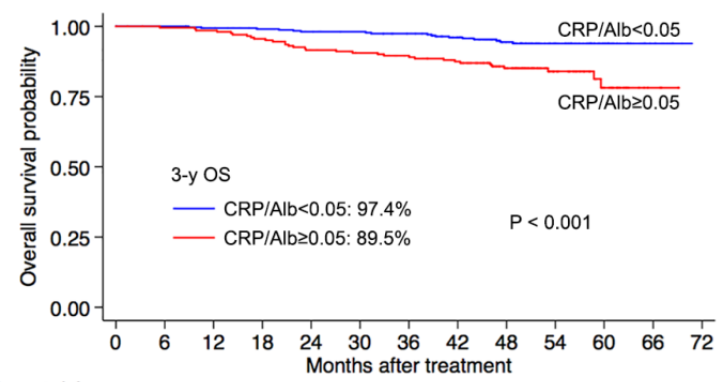

Number at ris

$\begin{array}{llllllllllllll}\text { CRP/Alb }<0.05 & 311 & 311 & 307 & 302 & 299 & 297 & 290 & 278 & 192 & 113 & 47 & 12 & 0\end{array}$

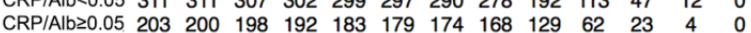

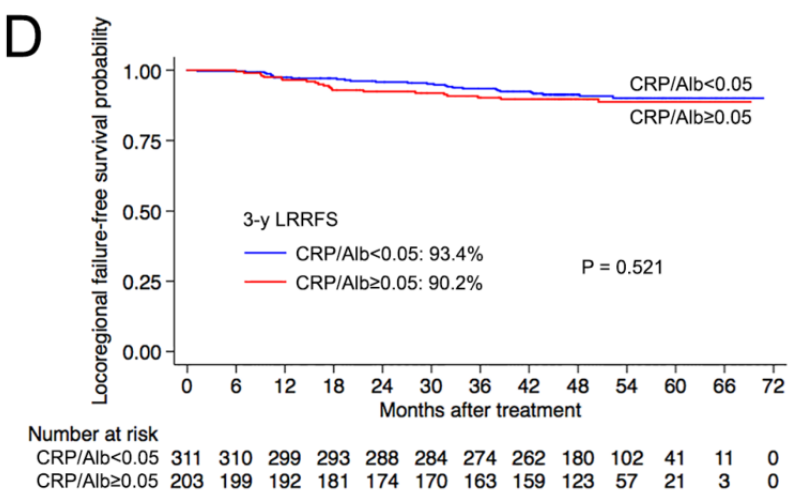

Figure 1: Kaplan-Meier overall survival (A), distant metastasis-free survival (B), locoregional relapse-free survival (C) and disease-free survival (D) curves for patients with a pretreatment CRP/Alb ratio $>0.05$ and $\leq 0.05$ in the training set. Abbreviations: $3-y=3$-year; CRP/Alb $=$ C-reactive protein/albumin; OS $=$ overall survival; DMFS = distant metastasis-free survival; LRRFS = locoregional relapse-free survival; DFS = disease-free survival.

A

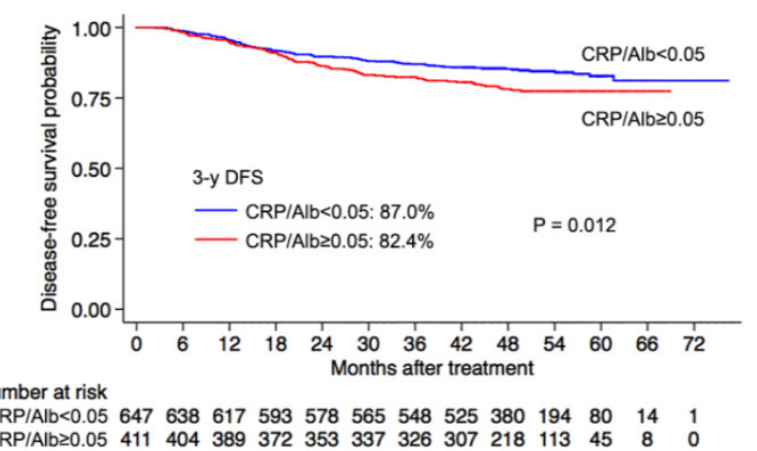

B

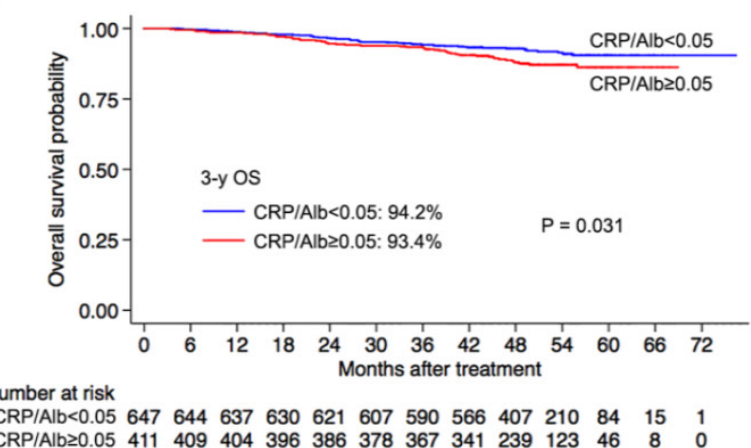

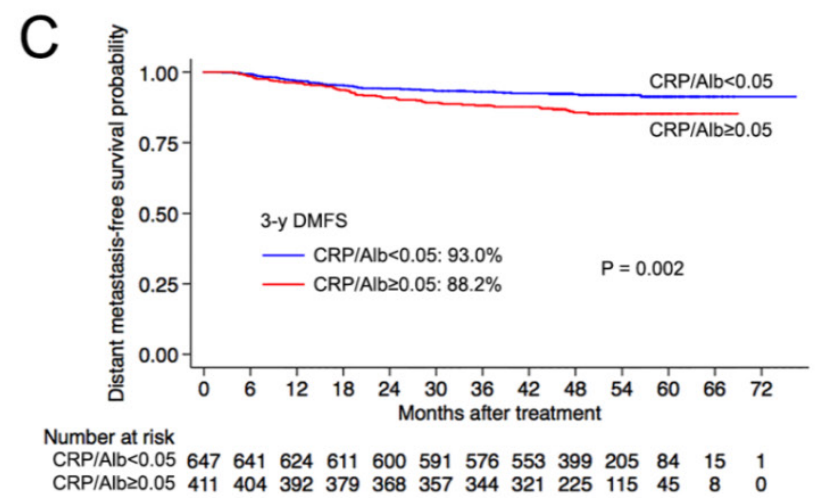

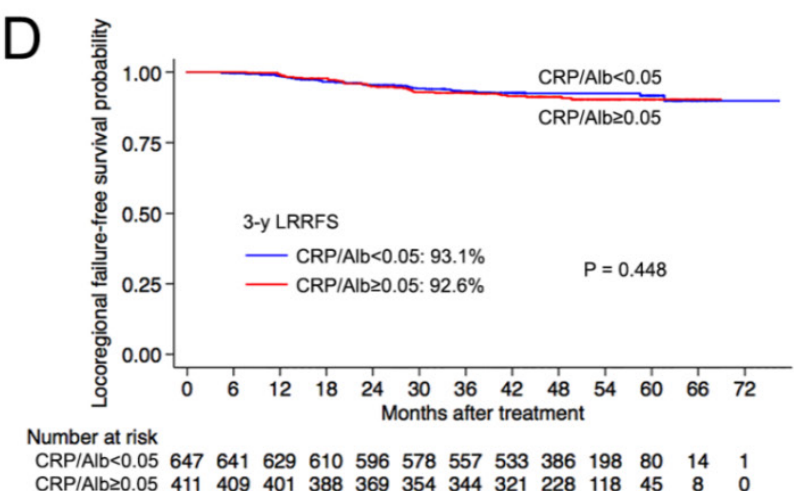

Figure 2: Kaplan-Meier overall survival (A), distant metastasis-free survival (B), locoregional relapse-free survival (C) and disease-free survival (D) curves for patients with a pretreatment CRP/Alb ratio $>0.05$ and $\leq 0.05$ in the validation set. Abbreviations: 3-y $=3$-year; $C R P / A l b=C$-reactive protein/albumin; OS $=$ overall survival; DMFS = distant metastasis-free survival; LRRFS = locoregional relapse-free survival; DFS = disease-free survival. 

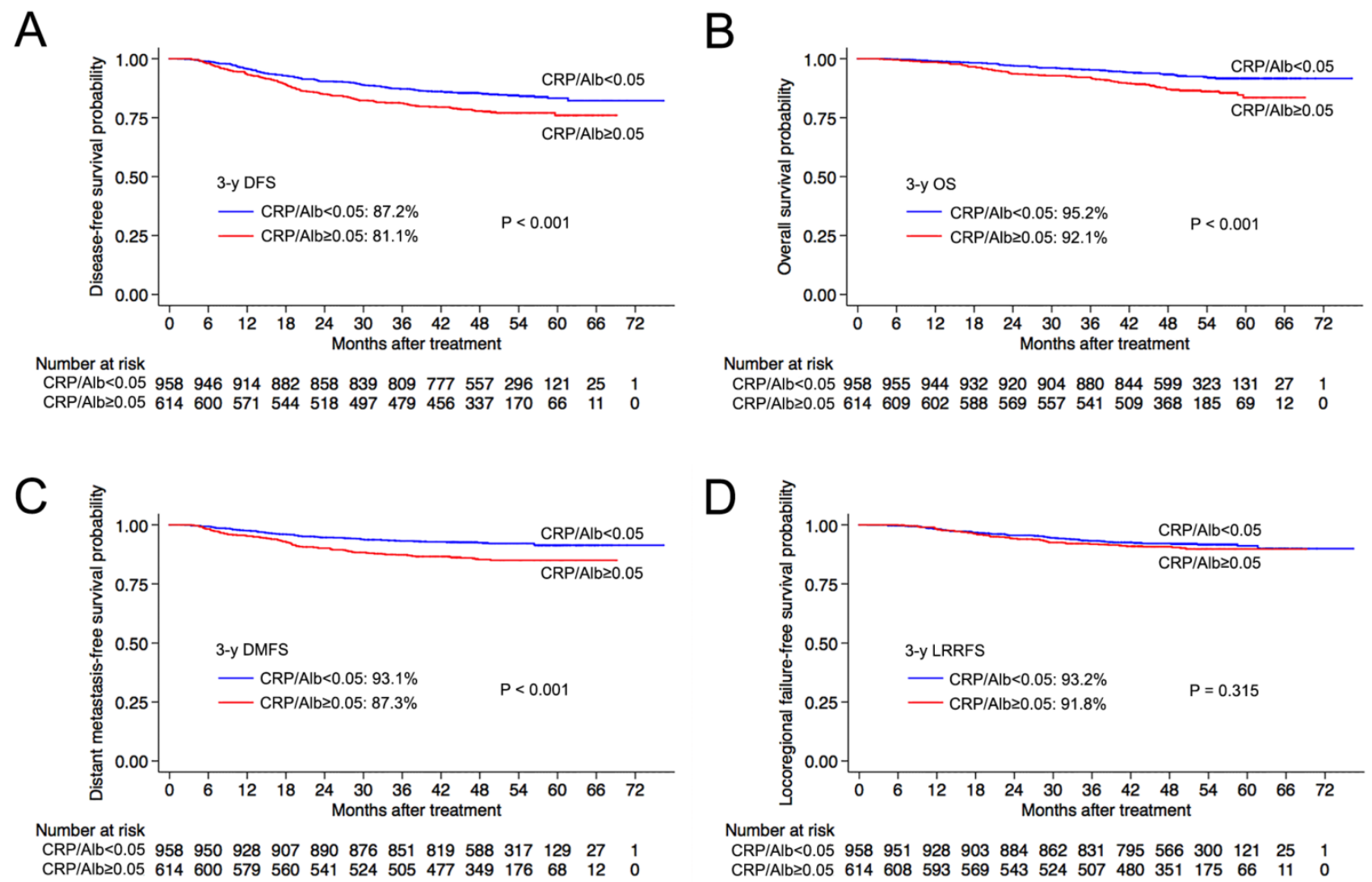

Figure 3: Kaplan-Meier overall survival (A), distant metastasis-free survival (B) and locoregional relapse-free survival (C) and disease-free survival (D) curves for patients with a pretreatment CRP/Alb ratio $>0.05$ and $\leq 0.05$ in the whole cohort. Abbreviations: $3-y=3$-year; CRP/Alb $=$ C-reactive protein/albumin; OS $=$ overall survival; DMFS = distant metastasis-free survival; LRRFS = locoregional relapse-free survival; DFS = disease-free survival.

\section{Comparison of the prognostic value of the pretreatment CRPIAlb ratio and $\mathrm{mGPS}$ in NPC}

Based on the criteria for the mGPS, only $4 / 1572$ $(0.3 \%)$ patients had both a high CRP level (CRP > 10 $\mathrm{mg} / \mathrm{L}$ ) and hypoalbuminemia (albumin $<35 \mathrm{~g} / \mathrm{L}$; mGPS = 2), 122/1572 (7.8\%) patients had a high CRP level but normal albumin (mGPS $=1$ ), and the remaining 1446 (91.9\%) patients had neither risk factor $(m G P S=0)$. In analysis of the entire cohort, the mGPS had significant prognostic value for 3-year OS (75.0\% vs. $90.1 \%$ vs. $94.4 \%, P=0.014)$, but no significant prognostic value for DMFS $(75.0 \%$ vs. $85.5 \%$ vs. $91.3 \%, P=0.141)$, LRRFS $(100.0 \%$ vs. $89.8 \%$ vs. $92.6 \%, P=0.565)$ or DFS $(75.0 \%$ vs. $77.9 \%$ vs. $85.4 \%, P=0.072$ ).

Multivariate analysis of the entire cohort was performed, incorporating host factors (age and gender), tumor factors (pathology type, $\mathrm{T}$ and $\mathrm{N}$ category), treatment method (radiotherapy alone vs. chemoradiotherapy), the pretreatment $\mathrm{CRP} / \mathrm{Alb}$ ratio and mGPS as covariates. The CRP/Alb ratio was confirmed as an independent prognostic factor for OS and DMFS, whereas the mGPS was not significant for any of these end points $(P=0.514$ and 0.789 , respectively).

\section{Discussion}

As the first analysis of the prognostic value of the $\mathrm{CRP} / \mathrm{Alb}$ ratio in NPC, this study indicates that the pretreatment $\mathrm{CRP} / \mathrm{Alb}$ ratio is an independent prognostic biomarker for overall survival and distant metastasis-free survival.

Inflammation is recognized to promote the initiation, progression and metastasis of cancer [15-19]. The major pathological type of NPC, undifferentiated non-keratinizing carcinoma - which accounted for $94 \%$ of the cases in this cohort - was formerly known as "lymphoepithelial carcinoma". This pathologic type contains prominent intermixed lymphocytes within the tumor, which indicates a possible role for inflammation in the development and progression of NPC. Serum CRP and albumin are proteins associated with the acute phase of inflammation. The change in proinflammatory cytokines might be the underlying mechanism of the progonostic value of the CRP/albumin ratio. For example, IL-6, a proinflammatory cytokine, plays an important role in the up-regulation of CRP during 
inflammation [26]. On the other hand, the overexpression of IL-6 was linked with lower albumin level [27]. Moreover, studies have demonstrated that IL-6 was able to promote carcinogenesis, tumor growth, and invasion in malignancies.[27-29] As a novel inflammation-based prognostic score, the $\mathrm{CRP} / \mathrm{Alb}$ ratio has been demonstrated to have prognostic value in hepatocellular carcinoma [20], small-cell lung cancer [30], esophageal squamous cell carcinoma [31] and gastric cancer [32]; the results of this study indicate that the pretreatment CRP/Alb ratio also has prognostic value in NPC.

Table 2. Summary of independent prognostic factors in multivariate analyses in the 1572 patients with NPC.

\begin{tabular}{|c|c|c|c|}
\hline Endpoints & Variables & HR (95\% CI) & $P$-value a \\
\hline \multirow[t]{13}{*}{ OS } & Age & $1.755(1.270-2.425)$ & 0.001 \\
\hline & $\mathrm{CRP} / \mathrm{Alb}$ ratio & 1.394(1.004-1.937) & 0.048 \\
\hline & $\mathrm{T}$ classification ${ }^{\mathrm{b}}$ & & $<0.001$ \\
\hline & $T 1$ & Reference & \\
\hline & $T 2$ & $3.095(1.313-7.297)$ & 0.010 \\
\hline & T3 & 3.077(1.404-6.742) & 0.005 \\
\hline & T4 & $6.136(2.765-13.619)$ & $<0.001$ \\
\hline & $\mathrm{N}$ classification $\mathrm{b}$ & & $<0.001$ \\
\hline & NO & Reference & \\
\hline & N1 & $1.723(0.886-3.352)$ & 0.109 \\
\hline & N2 & $2.876(1.404-5.889)$ & 0.004 \\
\hline & $N 3 a$ & 5.919 (2.307-15.187) & $<0.001$ \\
\hline & $N 3 b$ & 7.107 (3.452-14.630) & $<0.001$ \\
\hline \multirow[t]{12}{*}{ DMFS } & $\mathrm{CRP} / \mathrm{Alb}$ ratio & $1.545(1.124-2.122)$ & 0.007 \\
\hline & $\mathrm{T}$ classification ${ }^{\mathrm{b}}$ & & 0.018 \\
\hline & T1 & Reference & \\
\hline & $T 2$ & $1.295(0.697-2.406)$ & 0.412 \\
\hline & T3 & $1.229(0.728-2.074)$ & 0.436 \\
\hline & T4 & 2.055(1.178-3.583) & 0.011 \\
\hline & $\mathrm{N}$ classification $\mathrm{b}$ & & $<0.001$ \\
\hline & NO & Reference & \\
\hline & N1 & 2.047(1.054-3.979) & 0.035 \\
\hline & N2 & $4.123(2.045-8.315)$ & $<0.001$ \\
\hline & $N 3 a$ & 7.437(2.977-18.579) & $<0.001$ \\
\hline & $N 3 b$ & 7.195(3.479-14.883) & $<0.001$ \\
\hline \multirow[t]{13}{*}{ DFS } & Age & $1.302(1.023-1.657)$ & 0.032 \\
\hline & $\mathrm{CRP} / \mathrm{Alb}$ ratio & $1.235(0.973-1.568)$ & 0.083 \\
\hline & $\mathrm{T}$ classification ${ }^{\mathrm{b}}$ & & 0.001 \\
\hline & T1 & Reference & \\
\hline & $T 2$ vs $T 1$ & $1.445(0.910-2.294)$ & 0.118 \\
\hline & T3 vs T1 & $1.331(0.899-1.972)$ & 0.154 \\
\hline & $T 4$ vs $T 1$ & $2.164(1.423-3.292)$ & $<0.001$ \\
\hline & $\mathrm{N}$ classification $\mathrm{b}$ & & $<0.001$ \\
\hline & NO & Reference & \\
\hline & N1 & $1.948(1.241-3.059)$ & 0.004 \\
\hline & N2 & $2.926(1.785-4.798)$ & $<0.001$ \\
\hline & $N 3 a$ & $4.207(1.971-8.978)$ & $<0.001$ \\
\hline & $N 3 b$ & $4.985(2.952-8.420)$ & $<0.001$ \\
\hline
\end{tabular}

Abbreviations: HR, hazard ratio; CI, confidence interval; OS, overall survival; DMFS, distant metastasis-free survival; DFS, disease-free survival.

a $P$ values were calculated with an adjusted Cox proportional hazards model.

$\mathrm{b}$ According to the 7 th edition of the UICC/AJCC staging system.

The mGPS is another well-recognized inflammation-based prognostic score that is also based on the levels of CRP and albumin [33, 34]. However, the mGPS did not have significant independent prognostic value for any end-point in this cohort of patients. As only four (0.3\%) and 122 $(7.8 \%)$ patients had a mGPS of 2 and 1, respectively, and the remaining patients $(91.9 \%)$ had a mGPS of 0 , the mGPS cannot adequately distinguish patients with different levels of risk in NPC. Thus, the pretreatment CRP/Alb ratio may be more suitable for identifying patients at high risk than the mGPS.

This study has a number of limitations. Firstly, bias was unavoidable due to the retrospective nature of the analysis. However, the sample size was large ( $n$ $=1572$ ) and we randomly divided the cohort into the training set $(n=514)$ and validation set $(n=1058)$. The pretreatment $\mathrm{CRP} / \mathrm{Alb}$ ratio had similar prognostic value in the training set, validation set and the entire cohort. Secondly, this study only included patients treated at a single center in the endemic region. Further multi-center studies are warranted to confirm the prognostic value of the pretreatment CRP/Alb ratio in NPC. Additionally, the biological mechanisms that underlie the prognostic value of the CRP/Alb ratio in NPC are poorly understood and require further investigation.

In conclusion, the pretreatment $\mathrm{CRP} / \mathrm{Alb}$ ratio is a useful prognostic indicator in patients with NPC independent of disease stage, whereas the mGPS did not have independent prognostic value for any survival end-point. As assessment of both CRP and albumin are simple, routinely-available and standardized, the CRP/Alb ratio could serve as a convenient tool to improve prognostication for patients with NPC in the clinic. Future studies are warranted to investigate the mechanisms underlying the poor prognosis of patients with a high CRP/Alb ratio and develop effective interventions to improve the outcomes of these high-risk patients.

\section{Acknowledgments}

This work was supported by grants from National Science \& Technology Pillar Program during the Twelfth Five-year Plan Period (2014BAI09B10), Science and Technology Project of Guangzhou City, China (No. 14570006), the Planned Science and Technology Project of Guangdong Province (No. 2013B020400004) and Health \& Medical Collaborative Innovation Project of Guangzhou City, China (201400000001).

\section{Conflict of Interest}

The authors indicated no actual or potential conflicts of interest exist.

\section{References}

1. Chua ML, Wee JT, Hui EP, Chan AT. Nasopharyngeal carcinoma. Lancet. 2015. 
2. Wei KR, Zheng RS, Zhang SW, Liang ZH, Ou ZX, Chen WQ. Nasopharyngeal carcinoma incidence and mortality in China in 2010. Chinese journal of cancer. 2014; 33: 381-7.

3. Chan AT. Nasopharyngeal carcinoma. Annals of oncology : official journal of the European Society for Medical Oncology / ESMO. 2010; 21 Suppl 7: vii308-12.

4. Razak AR, Siu LL, Liu FF, Ito E, O'Sullivan B, Chan K. Nasopharyngeal carcinoma: the next challenges. European journal of cancer. 2010; 46: 1967-78.

5. Lee AW, Lin JC, Ng WT. Current management of nasopharyngeal cancer. Seminars in radiation oncology. 2012; 22: 233-44

6. Lee AW, Ng WT, Chan $\mathrm{YH}$, Sze $\mathrm{H}$, Chan C, Lam TH. The battle against nasopharyngeal cancer. Radiotherapy and oncology : journal of the European Society for Therapeutic Radiology and Oncology. 2012; 104: 272-8.

7. Lee AW, Sze WM, Au JS, Leung SF, Leung TW, Chua DT, et al. Treatment results for nasopharyngeal carcinoma in the modern era: the Hong Kong experience. International journal of radiation oncology, biology, physics. 2005; 61: $1107-16$

8. Wolden SL, Chen WC, Pfister DG, Kraus DH, Berry SL, Zelefsky MJ. Intensity-modulated radiation therapy (IMRT) for nasopharynx cancer: update of the Memorial Sloan-Kettering experience. International journal of radiation oncology, biology, physics. 2006; 64: 57-62.

9. Tham IW, Hee SW, Yeo RM, Salleh PB, Lee J, Tan TW, et al. Treatment of nasopharyngeal carcinoma using intensity-modulated radiotherapy-the national cancer centre singapore experience. International journal of radiation oncology, biology, physics. 2009; 75: 1481-6.

10. Lai SZ, Li WF, Chen L, Luo W, Chen YY, Liu LZ, et al. How does intensity-modulated radiotherapy versus conventional two-dimensional radiotherapy influence the treatment results in nasopharyngeal carcinoma patients? International journal of radiation oncology, biology, physics. 2011; 80: 661-8.

11. Ng WT, Lee MC, Hung WM, Choi CW, Lee KC, Chan OS, et al. Clinical outcomes and patterns of failure after intensity-modulated radiotherapy for nasopharyngeal carcinoma. International journal of radiation oncology, biology, physics. 2011; 79: 420-8.

12. Guo R, Sun $Y, Y u$ XL, Yin WJ, Li WF, Chen YY, et al. Is primary tumor volume still a prognostic factor in intensity modulated radiation therapy for nasopharyngeal carcinoma? Radiotherapy and oncology : journal of the European Society for Therapeutic Radiology and Oncology. 2012; 104: 294-9.

13. Zhang Y, Liu X, Zhang Y, Li WF, Chen L, Mao YP, et al. Prognostic value of the primary lesion apparent diffusion coefficient (ADC) in nasopharyngeal carcinoma: a retrospective study of 541 cases. Sci Rep. 2015; 5: 12242.

14. Zhou GQ, Tang LL, Mao YP, Chen L, Li WF, Sun Y, et al. Baseline serum lactate dehydrogenase levels for patients treated with intensity-modulated radiotherapy for nasopharyngeal carcinoma: a predictor of poor prognosis and subsequent liver metastasis. International journal of radiation oncology, biology, physics. 2012; 82: e359-65.

15. Balkwill F, Mantovani A. Inflammation and cancer: back to Virchow? Lancet. 2001; 357: 539-45.

16. Coussens LM, Werb Z. Inflammation and cancer. Nature. 2002; 420: 860-7.

17. Mantovani A, Allavena P, Sica A, Balkwill F. Cancer-related inflammation. Nature. 2008; 454: 436-44

18. Colotta F, Allavena P, Sica A, Garlanda C, Mantovani A. Cancer-related inflammation, the seventh hallmark of cancer: links to genetic instability. Carcinogenesis. 2009; 30: 1073-81.

19. Coffelt SB, de Visser KE. Cancer: Inflammation lights the way to metastasis. Nature. 2014; 507: 48-9.

20. Kinoshita A, Onoda H, Imai N, Iwaku A, Oishi M, Tanaka K, et al. The $\mathrm{C}$-reactive protein/albumin ratio, a novel inflammation-based prognostic score, predicts outcomes in patients with hepatocellular carcinoma. Annals of surgical oncology. 2015; 22: 803-10.

21. Edge SB, Byrd DR, Compton CC, Fritz AG, Greene FL, Trotti A. AJCC cancer staging manual (7th ed). New York, NY: Springer; 2010.

22. Chen $\mathrm{L}, \mathrm{Hu} \mathrm{CS}, \mathrm{Chen} \mathrm{XZ}, \mathrm{Hu} \mathrm{GQ}$, Cheng ZB, Sun Y, et al. Concurrent chemoradiotherapy plus adjuvant chemotherapy versus concurrent chemoradiotherapy alone in patients with locoregionally advanced nasopharyngeal carcinoma: a phase 3 multicentre randomised controlled trial. The Lancet Oncology. 2012; 13: 163-71.

23. Hanley JA, McNeil BJ. The meaning and use of the area under a receiver operating characteristic (ROC) curve. Radiology. 1982; 143: 29-36.

24. Zweig MH, Campbell G. Receiver-operating characteristic (ROC) plots: a fundamental evaluation tool in clinical medicine. Clinical chemistry. 1993; 39: $561-77$

25. Cox DR. Regression models and life- tables. J R Stat Soc Ser B (Methodological). 1972; 34: 187-220.

26. Pepys MB, Hirschfield GM. C-reactive protein: a critical update. J Clin Invest. 2003; 111: 1805-12

27. Kao JT, Feng CL, Yu CJ, Tsai SM, Hsu PN, Chen YL, et al. IL-6, through p-STAT3 rather than p-STAT1, activates hepatocarcinogenesis and affects survival of hepatocellular carcinoma patients: a cohort study. BMC Gastroenterol. 2015; 15: 50

28. Zhao G, Zhu G, Huang Y, Zheng W, Hua J, Yang S, et al. IL-6 mediates the signal pathway of JAK-STAT3-VEGF-C promoting growth, invasion and lymphangiogenesis in gastric cancer. Oncol Rep. 2016; 35: 1787-95.
29. Schneider MR, Hoeflich A, Fischer JR, Wolf E, Sordat B, Lahm H. Interleukin-6 stimulates clonogenic growth of primary and metastatic human colon carcinoma cells. Cancer letters. 2000; 151: 31-8.

30. Zhou T, Zhan J, Hong S, Hu Z, Fang W, Qin T, et al. Ratio of C-Reactive Protein/Albumin is An Inflammatory Prognostic Score for Predicting Overall Survival of Patients with Small-cell Lung Cancer. Sci Rep. 2015; 5: 10481.

31. $\mathrm{Xu}$ XL, $\mathrm{Yu} \mathrm{HQ}, \mathrm{Hu}$ W, Song $\mathrm{O}$ Mao WM. A Novel Inflammation-Based Prognostic Score, the C-Reactive Protein/Albumin Ratio Predicts the Prognosis of Patients with Operable Esophageal Squamous Cell Carcinoma. PloS one. 2015; 10: e0138657.

32. Liu X, Sun X, Liu J, Kong P, Chen S, Zhan Y, et al. Preoperative C-Reactive Protein/Albumin Ratio Predicts Prognosis of Patients after Curative Resection for Gastric Cancer. Transl Oncol. 2015; 8: 339-45.

33. Proctor MJ, Morrison DS, Talwar D, Balmer SM, O'Reilly DS, Foulis AK, et al. An inflammation-based prognostic score (mGPS) predicts cancer survival independent of tumour site: a Glasgow Inflammation Outcome Study. British journal of cancer. 2011; 104: 726-34.

34. Iwasaki Y, Ishizuka M, Kato M, Kita J, Shimoda M, Kubota K. Usefulness of an inflammation-based prognostic score (mGPS) for predicting survival in patients with unresectable malignant biliary obstruction. World J Surg. 2013; 37: $2222-8$ 